\title{
Estimation of Plasma Properties and Magnetic Field in a Prominence-like Structure as Observed by SDO/AIA
}

\author{
B. N. Dwivedi ${ }^{1}$, A. K. Srivastava ${ }^{2}$ and Anita Mohan ${ }^{1}$ \\ ${ }^{1}$ Department of Physics, IIT (BHU), Varanasi, India \\ email: bnd.app@iitbhu.ac.in \\ ${ }^{2}$ Aryabhatta Research Institute of Observational Sciences (ARIES), Nainital, India
}

\begin{abstract}
We analyze a prominence-like cool plasma structure as observed by Atmospheric Imaging Assembly (AIA) onboard the Solar Dynamics Observatory (SDO). We perform the Differential Emission Measure (DEM) analysis using various filters of AIA, and also deduce the temperature and density structure in and around the observed flux-tube. In addition to deducing plasma parameters, we also find an evidence of multiple harmonics of fast magnetoacoustic kink waves in the observed prominence-like magnetic structure. Making use of estimated plasma parameters and observed wave parameters, under the baseline of MHD seismology, we deduce magnetic field in the flux-tube. The wave period ratio $\mathrm{P} 1 / \mathrm{P} 2=2.18$ is also observed in the flux-tube, which carries the signature of magnetic field divergence where we estimate the tube expansion factor as 1.27 . We discuss constraints in the estimation of plasma and magnetic field properties in such a structure in the current observational perspective, which may shed new light on the localized plasma dynamics and heating scenario in the solar atmosphere.
\end{abstract}

Keywords. Solar Prominence, MHD Waves, Magnetic Fields

\section{Introduction}

In this brief paper, we report the differential emission measure (DEM) analysis using various AIA filters and deduce temperature structure of the emitting region using the method of Aschwanden et al. (2013). We also estimate local magnetic field in the tube from MHD seismology. We have carried out emission measure and temperature distribution analysis as well as tracing and estimating the density along the prominence by extensively using the automated method. SDO/AIA data provide information about the emission measure and estimation of the average density and temperature. We use the full-disk SDO/AIA in all EUV channels around 13:21 UT on 7 March 2011. We calibrate and clean the data using the aia_prep subroutine of SSWIDL and co-align the AIA images as observed in its various AIA filters using the co-alignment test. We obtain emission measure and temperature maps for six AIA filter full-disk and co-aligned images (304 $\AA, 171 \AA, 193 \AA, 94 \AA, 335 \AA$, and $211 \AA$ ) in the temperature between $0.4 \mathrm{MK}$ and 9.0 MK. The partial FOV analysis in the form of the emission measure and temperature measurements has been performed. The prominence structure is not fully resolved in multi-temperature emissions as it only contributes to the information of $304 \AA \mathrm{He}$ II emission. However, the cool plasma streaks are evident in the prominence sub-FOV maintained at temperature below 1.0 MK.

\section{Results}

The main results obtained are briefly described below:

[1] The automated emission analyses, using Aschwanden et al. (2013) method, clearly 
indicate the presence of cool plasma streaks in the prominence structure. The electron density decreases in the highly tangled prominence structure up to its apex which lies at a distance of $\approx 85 \mathrm{Mm}$ from the left-footpoint of the flux-tube. It shows some increment towards right foot-point side along the tube. The density near the apex of the prominence is $\mathrm{Ne}=1.0 \times 10^{9} \mathrm{~cm}^{-3}$. The density near the left foot-point lies in the range of $\mathrm{Ne}=$ $3.16 \times 10^{9}-1.0 \times 10^{10} \mathrm{~cm}^{-3}$.

[2] The temperature estimations with best least chi square show a uniform electron temperature $\left(\mathrm{Te} \sim 5.0 \times 10^{5} \mathrm{~K}\right)$ below $1.0 \mathrm{MK}$ along the prominence-like cool loop system. However, the calculated average temperature is $1.0 \mathrm{MK}$, because a few temperature points are estimated at $>1.0 \mathrm{MK}$. The average temperature of about $1.0 \mathrm{MK}$ is two orders of magnitude large than the cool prominence plasma temperatures (below $10^{4} \mathrm{~K}$ ) and most likely represent a very hot part of the prominence-corona transition region.

[3] Srivastava et al. (2013) observed the multiple harmonics of the fast magnetoacoustic kink waves in this prominence-like cool loop system.

[4] Srivastava et al. (2013) have found $\approx 667 \mathrm{~s}$ and $\approx 305 \mathrm{~s}$ periods respectively near the apex and foot-point of the prominence-like cool loop system. The phase speed of fundamental mode period (within tube length of $\sim 170 \mathrm{Mm}$ ) lies in the fast regime $\left(510 \mathrm{~km} \mathrm{~s}^{-1}\right)$ of the tube MHD waves, which can either be magnetoacoustic sausage or kink waves. However, under the given morphology and plasma conditions (typical coronal density inside the prominence), sausage modes are unlikely because of longer wavelength cut-off issue. Therefore, the most likely fast tubular mode is the non-linear magneto-acoustic kink waves that can weakly modulate the density near the boundary of the flux-tube (and thus intensity) during the modulation of plasma column depth in the obliquely oriented flux-tube in accordance with Cooper et al. (2003) theory.

[5] The period ratio shift of the fundamental mode to the first harmonics is $>2.0$, which indicates the flux-tube expansion and the magnetic field stratification in the loop. This is dominant over the longitudinal density structuring. For the first time, the expansion factor of the loop was estimated to be 1.27. It was also suggested as the first clues of the shift of fundamental mode period by a factor of 0.85 in the duration of $600 \mathrm{~s}$. This provides an observational clues to further develop the appropriate theory (Morton and Erdlyi, 2009 , Ruderman 2011).

[6] The average density near the apex of the observed filament is $\mathrm{Ne}=1.0 \times 10^{9} \mathrm{~cm}^{-3}$. Therefore, we estimate the magnetic field of the prominence near its apex as 9.0 Gauss based on the theory of MHD seismology and observational signature of the fundamental kink waves (Nakariakov and Ofman, 2001). Therefore, we estimate the density ( $\mathrm{Ne}=$ $\left.1.0 \times 10^{9} \mathrm{~cm}^{-3}\right)$, temperature $(\sim 1.0 \mathrm{MK})$, and magnetic field $(9.0$ Gauss $)$ values in the prominence-like flux-tube observed by SDO/AIA.

\section{Acknowledgements.}

We thank the referee for his/her suggestions. BNDs participation in the IAUS300 is enabled by the financial support from the ESA. We acknowledge SDO/AIANASA for data, and E. OShea for the randomlet software, and Torrence and Compo for the wavelet software. We also acknowledge the use of the procedures for automated DEM and Te - analyses developed by M.J. Aschwanden. AKS acknowledges the support from DST-RFBR and Indo-Austrian Projects (INT/RFBR/P-117;INT/AUA/BMWF/P-18/ 2013), and also thanks Shobhna Srivastava for her patient encouragement during research.

\section{References}

Aschwanden, M. J., Boerner, P., Schrijver, C. J., \& Malanushenko, A. 2013, ApJ, 283, 5 
Cooper, F. C., Nakariakov, V. M., \& Tsiklauri, D. 2003, A\&A, 397, 765

Morton, R. J. \& Erdélyi, R. 2009, ApJ, 707, 750

Nakariakov, V. M. \& Ofman, L. 2001, A\&BA, 372, 53

Ruderman, M. S. 2011, Sol. Phys., 271, 41

Srivastava, A. K., Dwivedi, B. N., \& Kumar, Mukul 2013, ApESSS, 345, 25 